\title{
Erratum to: Insights into the Tribochemistry of Silicon-doped Carbon-Based Films by Ab Initio Analysis of Water-Surface Interactions
}

\author{
Seiji Kajita ${ }^{1,2} \cdot$ M. C. Righi ${ }^{2}$
}

Published online: 15 March 2016

(C) Springer Science+Business Media New York 2016

\section{Erratum to: Tribol Lett (2016) 61:17}

\section{DOI 10.1007/s11249-015-0631-1}

The original version of this article unfortunately contained a spelling error in the article title. The word 'Insights' was incorrectly spelled as 'Insigths'. The error in the article title has been corrected with this erratum.

The online version of the original article can be found under doi:10.1007/s11249-015-0631-1.

M. C. Righi

mcrighi@unimore.it

Seiji Kajita

fine-controller@mosk.tytlabs.co.jp

1 Toyota Central R\&D Labs., Inc., 41-1, Yokomichi,

Nagakute, Aichi 480-1192, Japan

2 Istituto Nanoscienze, CNR-Consiglio Nazionale delle

Ricerche, 41125 Modena, Italy 